\title{
Load Balancing on the Internet
}

\begin{tabular}{|c|c|c|c|}
\hline \multicolumn{4}{|c|}{$\begin{array}{c}\text { Jianbin Wei, Wayne State University } \\
\text { Cheng-Zhong Xu, Wayne State University } \\
\text { Xiaobo Zhou, University of Colorado at Colorado Springs }\end{array}$} \\
\hline Introduction & 1 & Network-Side Load Balancing & 11 \\
\hline Workload Characteristics of Internet Services & 2 & Client State-Aware Load Balancing: Caching & \\
\hline Web Applications & 3 & Proxy Network & 11 \\
\hline Streaming Applications & 4 & Client and Server State-Aware Load Balancing: & \\
\hline Taxonomy of Load-Balancing Strategies & 4 & Content Delivery Network & 12 \\
\hline Load Balancing in the Server, the Network, and & & Client-Side Load Balancing & 14 \\
\hline the Client Sides & 4 & Server State-Blind Load Balancing & 14 \\
\hline State-Blind versus State-Aware Load Balancing & 5 & Server State-Aware Load Balancing & 14 \\
\hline Load Balancing at Different Network Layers & 5 & Summary & 14 \\
\hline Server-Side Load Balancing & 5 & Acknowledgment & 14 \\
\hline DNS-Based Load Balancing & 5 & Glossary & 14 \\
\hline Dispatcher-Based Load Balancing & 7 & Cross References & 15 \\
\hline Server Cooperative Load Balancing & 10 & References & 15 \\
\hline
\end{tabular}

\section{INTRODUCTION}

The explosive growth of the Web technology in the past decade created great opportunities for posting data and services on the Internet and making them accessible to the vast online users. Due to the unprecedented scale of the Internet, popular Internet services must be scalable to support up to millions of concurrent client requests reliably, responsively, and economically. These scalability and availability requirements pose great challenge on both processing power and networking communication capacity. Internet services have become an important class of driving applications for scalable computer systems. In particular, a cluster-based architecture is gaining momentum. The architecture deploys a cluster of networked server nodes that work collectively to keep up with ever-increasing request load and provide scalable Internet services, and load balancing is a key integration that distributes the client request load between the servers for scalable and highly available services. The architecture is often referred to as a Web cluster or a distributed Web server. This chapter gives a survey of state-of-the-art load-balancing strategies on the Internet.

Internet services are applications accessible to remote clients on the Internet via ubiquitous networking protocols. For scalability and availability, large-scale Internet services are often run on Web clusters. According to Hennessy and Patterson (2003), the Google search engine used more than 6,000 Linux/Intel PCs and 12,000 disks in December 2000 to serve an average of almost one thousand queries per second as well as index search for more than one billion pages. A most recent survey of large-scale Internet services also revealed that all the representative services were running on a cluster of hundreds or even thousands of servers at more than one data centers (Oppenheimer \& Patterson, 2002). Internet services on a Web cluster rely on the load-balancing technology to

distribute client requests between the servers efficiently and transparently.

The concept of load balancing is not new. It has long been developed as a prominent technology in both distributed systems and parallel computers. However, load balancing on the Internet distinguishes itself by its new Internet workload characteristics and unique quality of service (QoS) objectives. In parallel computers, load balancing is to assign parallel tasks of a job onto (virtual) processors with the objective of balancing the processors' workload and meanwhile minimizing the interprocessor communication cost due to the task dependences (Xu \& Lau, 1997). Its overall goal is to reduce the execu(Xu \& Lau tion time of parallel tasks. In traditional distributed systems, load balancing is to schedule the execution of independent jobs with the objective of efficient utilization of system-wide computational resources. It is often implemented in a form of load sharing-ensuring no processor is idle while there are jobs waiting for services in other processors.

By contrast, load balancing on the Internet is to distribute the Web service requests between the client, server and network for enhancing the level of QoS in terms of the service responsiveness, scalability, and availability Figure 1 shows a typical information flow infrastructure of the Web services. To access Internet service via a URL address like http://www.yahoo.com, which contains the domain name www.yahoo.com, a client first contacts its local domain name system (DNS) to get the IP address of the domain name. If its local DNS does not contain the IP address, the domain name resolution request goes upward to the root name server until the service's authoritative DNS is reached. The resolved IP address of the domain name is usually stored in the local DNS for reuse in the future. With the IP address, the client sends subsequent service requests to the server. The requests may go through a client proxy, Internet service provider (ISP) 


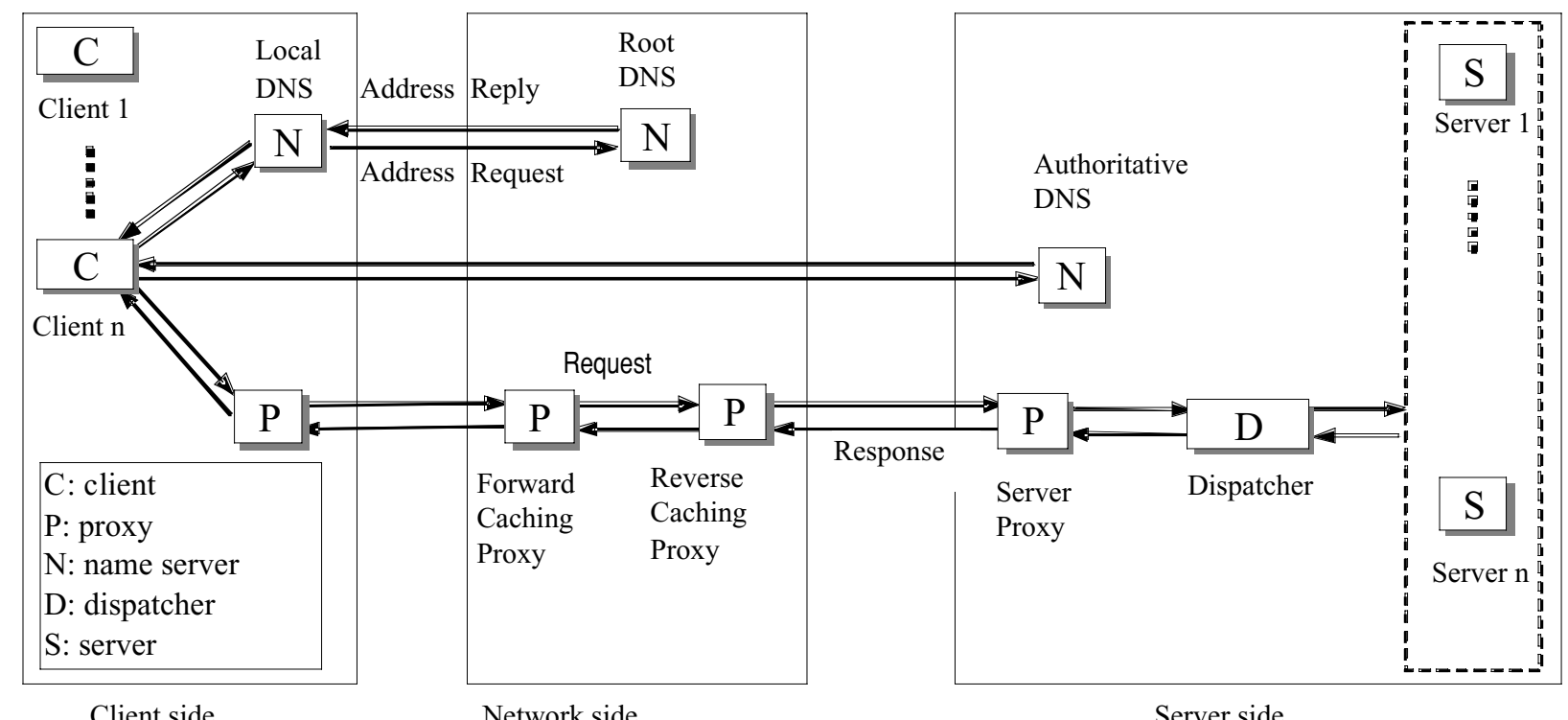

Figure 1: A typical information flow infrastructure of an Internet service.

proxies in the network, and a server proxy. The IP address may be a virtual IP address of a Web cluster. In this case, it is the dispatcher in front of the cluster that decides the actual server in the back-end to serve the incoming requests.

Two primary performance metrics related to QoS are capacity and latency. Capacity refers to the number of concurrent requests that the server can support without causing significant queueing delay. Latency is the service time, as perceived by the client. It is the request processing time in the server, including its possible queueing delay, plus the request/response transmission time in the network. These two metrics are related. A high-capacity server would cause less queueing delay and consequently lead to quick responses to the requests.

For the objective of QoS, load-balancing techniques on the Internet usually make use of multiple servers to process the client requests in parallel. The servers can be either distributed locally at the same site or globally distributed at different geographical locations. We refer to the techniques on locally distributed servers as server-side load balancing (or server load balancing) and the techniques on globally distributed servers as networkside load balancing (or network load balancing). Serverside load balancing aims at scaling the server processing capacity and communication bandwidth with the increase of the number of participating servers. Due to the scale of the Internet, service transmission time on the network is a significant performance factor, in particular in streaming applications. Wide deployment of broadband networks helps alleviate the problem to some extent. On the other hand, fast networking entices service providers to create services eager for more bandwidth. Network-side load balancing is to move the services closer to clients so as to reduce the network transmission delay on the Internet.

Load balancing on the Internet is also concerned about the workload distribution between servers and the clients.
For example, e-commerce services often use mobile codes like JavaScript to verify integrity and validity of user inputs at the client side. This not only avoids back-and-forth data transmission between the server and the client but also reduces the server-side processing workload. We refer to this type of load balancing as client-side load balancing (or client load balancing). A primary objective of client-side load balancing is to improve the quality of Internet services by migrating certain tasks from server to client. Recent advances in peer-to-peer computing further the concept of client-side load balancing by allowing ther te high

Load-balancing mechanism aside, another important aspect of load balancing is policy. Internet services exhibit different workload characteristics from traditional parallel and distributed applications. Unlike jobs in distributed systems and tasks in parallel computing, the basic scheduling unit of load balancing on the Internet is client request. The requests are nonuniform in size because they may need to access different data and cause different types of operation. Although the requests from different clients are independent, they are actually related if they are about to access the same data. The resion and must be handled together. Due to these distinct workload characteristics, many different policies of load balancing have been developed. This chapter surveys representative policies under different load-balancing mechanisms. It starts with a description of Internet traffic characterization, followed by a classification of load-balancing strategies.

\section{WORKLOAD CHARACTERISTICS}

\section{OF INTERNET SERVICES}

Load balancing on the Internet is largely determined by workload characteristics of the Internet services. Two 
important Internet services are Web and streaming applications.

\section{Web Applications}

Web traffic, carried by HTTP protocol over TCP, is one of the dominant components of Internet traffic. It is closely tied to contents of Web pages and to dynamics of the TCP transmission protocol. Past studies of Web workloads TCP transmission protocol. Past studies of Web workloads
concentrated on conventional information provider sites and found several important characteristics common to the sites: target file types, file size distributions, file popularity distributions, self-similarity in Web traffic, reference locality, and client request patterns. An early study summarized the Web traffic characteristics as follows (Arlitt \& Williamson, 1997):

- Images and HTML files together account for over $90 \%$ of the files transferred. The majority of HTTP requests for Web pages are smaller than 500 bytes. HTTP responses are typically smaller than 50 Kbytes and the median transfer size is small (e.g., less than 5 Kbytes).

- Ten percent of the files account for $90 \%$ of requests and bytes transferred. The file popularity distributions are Zipf-like. The file size distributions are heavy-tailed. The file transfer size distributions are also heavy-tailed, although not as heavy-tailed as the file size distributions.

- The user request patterns are exponentially distributed and independent (e.g., Poisson distributions). However, significant traffic variance (burstiness) is present on a wide range of time scales. The aggregate traffic generated by many users of the Web has been shown to exhibit self-similarity.

The characteristics of Web traffic have a significant impact on choosing load-balancing strategies. It is often assumed that the service time of a request is proportional to the size of its requested file. Simple strategies, like random and round-robin algorithms, are good enough when the requests are uniform in size and independent (Kwan, McGrath, \& Reed, 1995). To take advantage of temporal McGrath, \& Reed, 1995). To take advantage of temporal
locality in requests, locality-aware distribution strategies locality in requests, locality-aware distribution strategies et al., 1998). In the case that the file transfer size distribution is heavy-tailed, a size interval task assignment with equal load (SITA-E) performs excellent in terms of both mean response time and mean slowdown, provided the system load is not too high (Harchol-Balter, Crovella, \& Murta, 1999)

Recent years witnessed a number of major changes in the Web applications (Arlitt, Krishnamurthy, \& Rolia 2001; Padmanabhan \& Qiu, 2000). The most notable ones include the following: Web pages are shifting from static content to dynamic content; e-commerce becomes one of the major Web applications; and continuous media are inthe major Web applications; and continuous media are inerated by programs that run on servers every time the corresponding pages are accessed for producing a different page for every access. Simple examples are the pages that contain displays of a visitor counter or the curren date and time. Responses of search engines, stock-quote sites, and personalized Web pages, which are generated on the fly from databases, are typical examples of dynamic pages. Although dynamic pages provide a far richer experience for users than static pages, generating dynamic pages on the fly imposes additional overhead on server resources, especially on CPU and disk I/O. These changes of the workload characteristics pose a challenge to existof the wo existing load-balancing techniques in performance. Some of the strategies are even no longer applicable. For example, a size-based strategy may not work for dynamic contents because the service time for dynamic content due to its size is unknown. The potential for caching requested files declines and some requested files are even noncacheable because they cause dynamic page generations.

Interest in e-commerce has grown substantially in the past several years (Arlitt et al., 2001; Menasce, Almeida, Fonseca, \& Mendes, 1999). E-commerce workloads are composed of sessions. A session is a sequence of requests of different types made by a single customer during a single visit to a site. During a session, a customer can issue consecutive requests of various e-commerce functions such as browse, search, add to the shopping cart, register, and pay. Different customers may exhibit different navigational patterns and hence may invoke the differen functions in different ways and with different frequencies. Menasce et al. (1999) present analysis techniques of workload and performance modeling as well as capacity planning for e-commerce sites.

The key QoS issues on e-commerce sites include responsiveness, availability, and security. For responsiveness and availability, it is increasingly common for ecommerce sites to include the capacity of load balancing to avoid bottlenecks or overloading servers. Loadbalancing strategies must be session-oriented. That is, they must direct all consecutive and related requests of a session from a given customer to the same server. This is known as session integrity (also referred to as sticky connections). For example, a customer may add an item to the shopping cart over a TCP connection that goes to server 1 . If the next connection goes to server 2 , which does not have the shopping-cart information, the application breaks. This required coordination in serving of related requests is referred to as client affinity (affinity routing). Hence, the round-robin load-balancing strategies are not applicable in the stateful e-commerce applications while locality-aware request routing mechanisms provide a convenient way to support session integrity.

For security, e-commerce transactions are often encrypted via the secure socket layer (SSL) protocol. Processing SSL transactions puts an extra load on server resources. This means customer's transaction requests and browsing requests have different requirements for the server-side resources and different impacts on the revenue of the sites. On the other hand, an e-commerce merchan tends to share servers handling transaction requests with ther merchants in a Webcluster. Load-balancing st other merchants in a Web cluster. Load-balancing strategies must deal effectively with these issues. For exam-
ple, Wolf and Yu (2001) proposed a family of load- balancing algorithms to dispatch various types of customer requests to different servers. The servers are partitioned into two categories, sharable for the public requests (such as browse requests) and not sharable for private requests (such as transactions). 


\section{Streaming Applications}

The Internet is seeing the gradual deployment of streaming applications, such as audio over IP, video conferencing, and video-on-demand (VoD). These streaming applications generate traffic with characteristics and requirements that differ significantly from traffic generated by conventional Web applications.

Audio and video streams are continuous and timebased. The term "continuous" refers to the user's view of the data. Internally, continuous streams are represented as a sequence of discrete elements (audio sample, video as a sequence of discrete elements (audio sample, video
frame) that replace each other over time. They are said to be time-based (or isochronous) because timed data elements in audio and video streams define the semantics or "content" of the streams. Timely delivery of the elements is essential to the integrity of the applications. Hence, streaming application quirements. However, unlike typical Web pages, streaming media does not necessarily require the integrity of the objects transferred. There can be some packet loss, which may simply result in reduced quality perceived by users.

Continuous streams are often bulky in transmission. This is especially so for video of a reasonable quality. Media servers that support streaming applications need to move data with greater throughput than conventional Web servers do. Typical audio stream rates remain on the order of tens of kilobytes per second, regardless of the encoding scheme. Video streams span a wide range of data rates, from tens of kilobytes per second to tens of megabytes per second. The server disk-I/O and networkI/O bandwidth can be the resource bottleneck. The charI/O bandwidth can be the resource bottleneck. The char-
acteristics of encoded video (frame size and frequency) vary tremendously according to the content, the video compression scheme, and the video-encoding scheme. In addition, streaming traffic usually consists of a control part and a data part, potentially using different protocols like RTSP and VoIP, while conventional Web traffic is homogeneous in this case. Hence, a traffic manager must parse the control channels to extract the dynamic socket numbers for the data channels so that related control and data channels can be processed as a single, logical session.

Compared to the studies of Web workload characterization, few insightful studies of streaming workloads exist. Past studies of streaming workloads found several common characteristics (Chesire, Wolman, Voelker, \& Levy, 2001):

- The video popularity distributions are Zipf-like. The skew parameter $a$ ranges from 0.271 to 1.0

- Request arrival patterns are nonstationary. For example, in some movie-on-demand systems, the rush hour is usually around $9 \mathrm{PM}$.

- Users tend to preview the initial portion of a video to find out whether they are interested.

These characteristics and requirements of streaming applications impose a great impact on caching and load balancing. For example, the video-browsing pattern suggests that caching the first several minutes of video data should be effective. However, this partial cache notion is not valid for Web object requests. The large video size and the typical skews in video popularity suggest that several requests for the same object coming within a short time scale be batched and a single stream be delivered to multiple users by multicast so that both the server disk I/O and network bandwidth requirements can be disk $\mathrm{I} / \mathrm{O}$
reduced.

Due to the media popularity distributions, data placement methods can also be crucial to load balancing in distributed streaming servers. There are essentially two complementary techniques: striping (i.e., data partitioning between servers) and replication. The main advantages of data striping are high disk utilization and good load-balancing ability. However, a wide-range data striping can lead to high scheduling and system expansion overhead. The cost is especially high when the servers are to be deployed in a geographically distributed environment. Replication tends to isolate servers from each other for scalability and reliability. Nevertheless, it can lead to load imbalance produced by the uneven distribution of media popularity, because the servers storing hot media files can be overwhelmed by client requests.

\section{TAXONOMY OF LOAD-BALANCING}

\section{STRATEGIES}

Internet services are applications that are, as shown in Figure 1, realized through collaborations between three parties: the service provider (i.e., server), the delivery network, and the service requester (client). Accordingly, loadbalancing techniques can be deployed in each of these three sides to distribute and deliver the client request load between the participating components in the information flow path. Load-balancing strategies can also be classified according to the type and amount of information about the flow path they take into account. The third categorization dimension of load-balancing strategies is their implementation layer in the network protocol stack.

\section{Load Balancing in the Server, the Network, \\ and the Client Sides}

A scalable Internet service is often provided by a group of servers either connected by a local area network (LAN) or distributed over a wide area network. We refer to the locally distributed servers that collaboratively provide the same Internet services on a single location as a Web cluster. Load balancing in a Web cluster focuses on the distribution of client requests between its servers for the purpose of increasing the service responsiveness, scalability, and availability. Three major distribution approaches exist: DNS-based, dispatcher-based, and cooperative server based. Since all Internet services are advertised by their URLs, which include their domain names, the DNS-based approaches rely on the server-side authoritative DNS server to make load-balancing decisions during the process of domain name resolutions. This process allows the authoritative DNS server to implement different load-balancing policies to select an appropriate server for each client request. The DNS-based approach 
can be easily scaled from a Web cluster to globally distributed servers and there would be no bottleneck risk in the DNS server. However, the DNS-based approach can only control a small fraction of address-mapping requests due to the presence of caching in the information flow path and cannot directly control subsequent retion flow path and and other name servers along the path between the client and the authoritative DNS server. Moreover, although the authoritative DNS server can detect failed servers and exclude them from the list of available servers, clients may still send them requests because of the cached IP addresses.

By contrast, the dispatcher-based approach relies on a load-balancing dispatcher in front of the Web cluster to distribute the client requests. However, the dispatcher itself can be a potential performance bottleneck, especially when it needs to process all responses from the servers This centralized load-balancing strategy is mostly applicable to Web clusters and is hardly extensible to wide area network environments.

The cooperative server-based approach uses a twolevel routing mechanism, in which the authoritative DNS server initially distributes client requests to servers based on a simple policy like random or round robin. On the receipt of a request, a server may handle it by itself or redirect the request to another one according to the states of the servers. Unlike the dispatcher-based approach, this of the servers. Unlike the dispatcher-based approach, this
kind of approach allows all the servers to participate in making decisions on load balancing. It avoids single failure points and potential performance bottleneck and offers good scalability. On the other hand, request redirections may cause an increase of response time in certain situations.

The objective of load balancing on the network side is to reduce the service delivery overhead by deploying server replicas (or surrogates) close to its prospective clients. Network-side load-balancing techniques can be integrated with caching proxy servers and content delivery networks (CDN). The caching proxy servers store $\mathrm{Web}$ resources in anticipation of future requests. It therefore can reduce the network transmission latency and servercan reduce the network transmission latency and server-
side overhead. With the support of CDN, service providers side overhead. With the support of CDN, service providers
duplicate their services in geographically distributed surrogates around the world (network edge). On the receip of a client request, the CDN makes use of DNS-based load-balancing strategies to direct the request to the most appropriate surrogate according to the client geographappropriate surrogate according to the client geograph-
ical locations, the CDN topological information, states ical locations, the CDN topological information, states
of the servers, etc. Therefore, DNS-based approaches are also included in the network-side load-balancing category.

On the client side, there are also load-balancing techniques intended to relieve the server workload incurred by the client requests. To access a service provided by more than one server, a smart client can direct its requests to an appropriate replica if the client has some state information about the replicas. In e-commerce services, mobile codes like JavaScript are widely used to verify integrity and validity of the client inputs at the client side to avoid the back-and-forth communications between the serv and its clients.

\section{State-Blind versus State-Aware \\ Load Balancing}

The second characteristic of load-balancing strategies is the state information considered by the strategies. The state information about a client request includes the clien IP address, port number, physical location, request content, request priority, and round-trip time between the client and the server Client state information like session ID and cookies facilitates client-affinity scheduling to ensure that the requests from the same client in a session are handled by the same server in a Web cluster. The state information about a server includes its static configuration and dynamic workload (e.g., the number of active connections and response times).

State-blind policies assume little knowledge about the state information, except static configuration parameters. Since they do not rely on content-specific information or dynamic workload information about the servers, their implementations incur little run-time overhead. However the state-blind policies may make poor assignment decisions due to highly variable client requests and the change of server workloads. State-aware policies, in contrast, tend to make adaptive decisions in response to the request content information and/or the workload change. The state-aware policies can be further classified into three categories: client state aware, server state aware, and client and server state aware.

\section{Load Balancing at Different Network Layers}

The third classification dimension of the load-balancing strategies is the network layer of their implementations. This implementation issue has a great impact on the performance and efficiency of load-balancing strategies because it determines the possible information to be used by the strategies and the related overhead. Two major implementation strategies are layer-4 (TCP layer) and layer-7 mentation strategies are layer-4 (TCP layer) and layer-7 (application layer) implementation with respect to the OSI network stack.

In a layer-4 implementation, information like IP address and TCP port number can be used to select a server for each request. However, it is limited to content-blind load-balancing policies because this approach cannot get any application-specific information in this layer. By contrast, a layer-7 implementation can take full advantage of the information obtained from the lower layers. For example, a session identifier can be used for session affinity scheduling in e-commerce services; request-specific contents from the application layer can be used for contentaware scheduling.

In summary, we present our classification of the loadbalancing strategies in Figure 2. It also contains representatives of each category in both academia and industry. Details of the strategies are reviewed in the next three sections.

SERVER-SIDE LOAD BALANCING DNS-Based Load Balancing

Recall that an Internet service is often advertised by its URL. As shown in Figure 1, a domain name resolution 


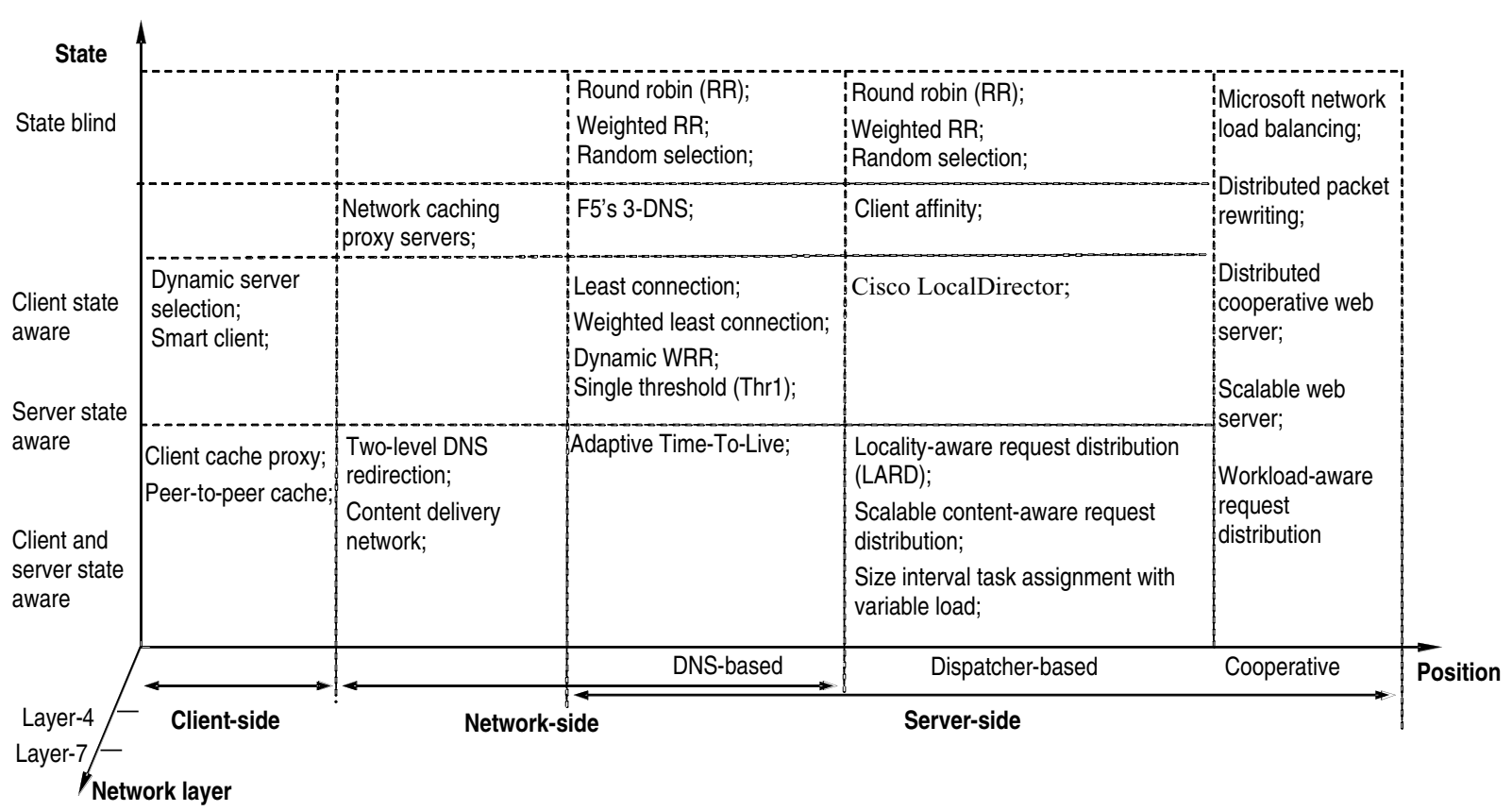

Figure 2: Taxonomy of load-balancing strategies on the Internet.

is carried out by the DNS servers along the path from the client browser to the service authoritative DNS server. DNS-based load-balancing strategies rely on the authoritative DNS server to map the domain name to an appropriate server IP address for each client request. To propriate server IP address for each client request. To reduce the response time for future requests, the resolu-
tion is usually cached on the intermediate DNS servers and browser cache. Each resolution has a property of time-to-live (TTL) set by the authoritative DNS server. It determines a valid period of the resolution in the caches.

\section{State-Blind Load Balancing}

The simplest DNS-based load-balancing approach in a Web cluster is round robin (Kwan et al., 1995). In this approach, the DNS server assigns the IP addresses of the cluster servers in a round-robin manner to the clients' address-mapping requests. It uses a circular server list and a pointer to the last selected server to make the decision. That is, if the last assignment was to server $S_{i}$, then the current request will be assigned to $S_{(i+1) \bmod n}$, where $n$ is the number of servers. Random assignment is another simple state-blind approach, in which the DNS server assigns requests to the servers randomly. Statistically, given a large number of requests, this approach can guarantee an equal distribution of client requests to the available server IP addresses.

The round-robin approach can be easily extended to clusters with heterogeneous servers. A variant of the round-robin policy is weighted round robin (WRR). It assigns requests to the servers according to the relative weights of the server capacities. The weights can be calculated in terms of static server configuration information like CPU speed, memory size, and network bandwidth. We refer to this as static WRR.

\section{Client State-Aware Load Balancing}

The client state-blind approaches can be extended to take into account some client-specific information in loadbalancing decisions. F5's 3-DNS Controller (F5 Network, n.d.) implemented a strategy based on proximity-based address mapping for geographically distributed servers. By comparing the IP address of the client with the IP addresses of the available servers, the controller can determine the proximity of the servers and assign the client's requests to the nearest server.

\section{Server State-Aware Load Balancing}

There are also DNS-based approaches that utilize serverside state information for load balancing. A leastconnection policy allows the DNS server to assign requests to the server with the least number of active connections. The server workload information can be either periodically reported by the servers or polled by the DNS server on demand. A variant of the least-connection policy is weighted least-connection policy for clusters with heterogeneous servers. Denote $W_{i}$ and $C_{i}$ as the server weight and the number of active connections, respectively. In the weighted least-connection policy, the DNS server will assign the next client request to the server with minimal $C_{i} / W_{i}$.

Another example of the server state-aware policies is dynamic WRR. In this policy, the DNS server calculates the server weights periodically in terms of their dynamic workload such as the number of active connections or measured response time, and assigns each incoming request to a server in the same way as WRR. A critical question is how often does the DNS server need to collect the server workload information? An alternative to periodical workload information collection is an asynchronous alarm mechanism, in which the servers keep evaluating 


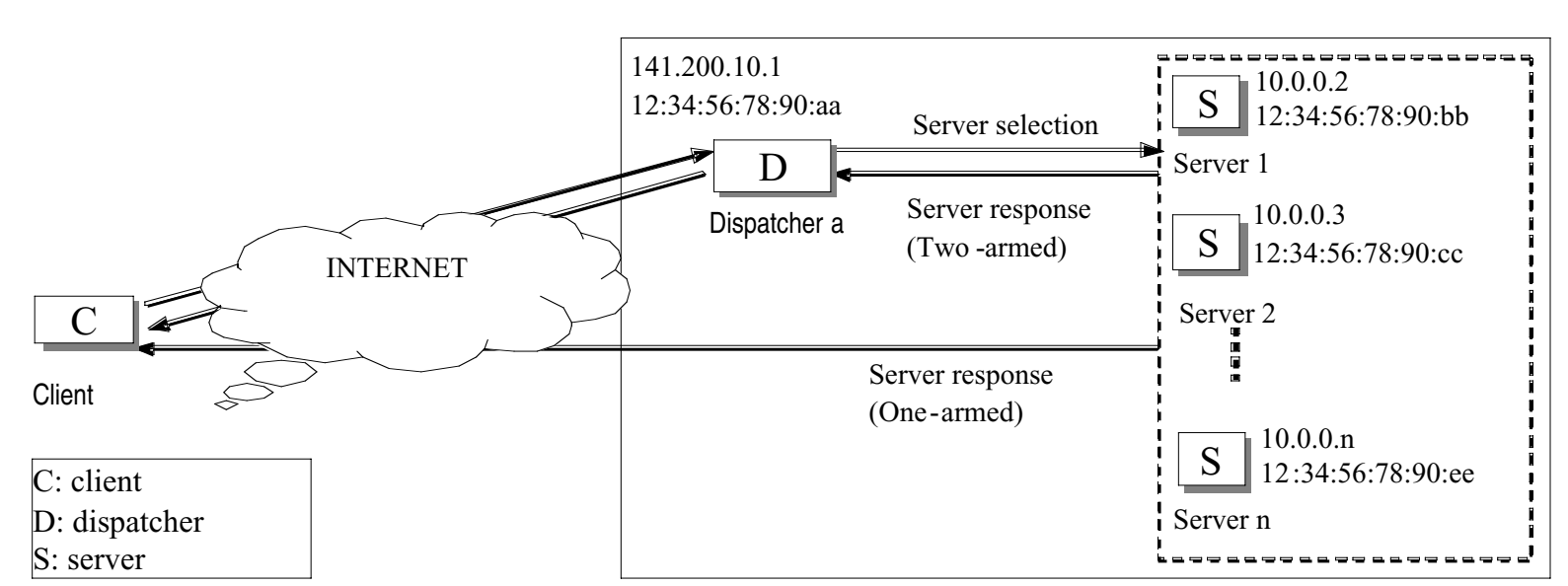

Server side

Figure 3: A dispatcher-based load-balancing infrastructure.

their resource utilization and send the DNS server alarms only if their utilization has exceeded a predefined load threshold. The DNS server excludes any alarmed server from the available server list until the server load is back to normal.

\section{Client and Server State-Aware Load Balancing}

There are DNS-based load-balancing approaches that take into account the state information about both sides. An example is adaptive TTL (Colajanni, Yu, \& Cardellini, 1998). It uses hidden load weight, which is the number of incoming requests from different client domains, to distribute requests. The DNS server assigns client requests to the available servers in the round-robin manner and associates a client-specific TTL value to each request. The client-specific TTL is set in terms of the hidden load weight of the client domain as well as the server processing capacity. It is known that the hidden load weight increases with the TTL value. The TTL should be set inversely proportional to the hidden load weight in order to amortize the hidden loads from different client domains while being proportional to the server processing capacity.

In summary, the DNS-based load-balancing approaches are simple to implement because they do not require modifications of the existing network switch. Their main drawbacks are

- Unbalanced load distribution. As we see from Figure 1 the DNS-based approaches allow clients to contact the server directly based on their cached name resolutions. This is prone to unbalanced load distribution between the servers. Although the authoritative DNS can set the TTL value to limit the duration of each resolution in concept, it will not take effect without the cooperation of intermediate DNS servers and the client's local DNS server. In addition, a small value of TTL would lead to a perver. In addition, a small value of TTL would bottleneck in the authoritative DNS.

- Low availability. Due to the existence of name resolutions in caches, client requests will continue to be sent to a server, whether the specific server is online or not, before the resolution expires. Consequently, some clients may perceive a service failure, even though there are other servers available for the service.

It is because of these drawbacks that DNS-based approaches became rarely used for load balancing in locally distributed Web clusters. However, they can be tailored to direct requests to geographically distributed servers for global load balancing. Related issues in the context of network-side load balancing will be reviewed in NetworkSide Load Balancing.

\section{Dispatcher-Based Load Balancing}

In a Web cluster, a dispatcher serves as the cluster gate, as shown in Figure 3, which intercepts all incoming requests. It has a single public IP address representing the Web cluster. Since the Web cluster together with the dispatcher form a virtual server, the IP is often referred to as virtual IP (VIP) address of the virtual server. The dispatcher identifies each server in the cluster with a unique private address, either an IP or a media access address (MAC) address, depending on the architecture. In contrast to the DNS server, the dispatcher has complete control over the routing of all incoming requests and acts as a centralized scheduler. It selects the most appropriate server according to a load-balancing policy and forwards the requests to the selected server.

The choice of the routing mechanism has significant impact on the performance and efficiency of the strategies because different routing implementations will reveal different amounts of information about the client requests at different costs. Two major implementations are the network layer (layer-4) and the application layer (layer 7) with reference to the OSI network stack. Layer-4 implementations are oblivious to the client request content. They simply forward the client requests to a selected server regardless with the requests are connection requests or not. Layer-7 implementations distinguish connection establishment requests from those following up requests in a connection. On the receipt of a client connection request, the dispatcher establishes a TCP commu- 
requests from the channel and relaying them to the servers selected based on a load-balancing policy.

The dispatcher can be configured into two functional modes according to the way in which the response packets are routed: two-way (two-armed) and one-way (onearmed). The one-way configuration allows servers to return their responses directly to the clients, without going through the dispatcher. In contrast, the two-way configuration requires all the responses to pass through the dispatcher. Therefore, it allows different subnet configuration for the servers. There are many different ways to route packets at the network layer in each configuration.

\section{Two-Way Dispatcher Architecture}

Packet rewriting is a layer-4 implementation based on the IP network address translation (NAT) (Egevang \& Francis, 1994). In a Web cluster, the dispatcher is set to a different IP address from the servers and configured as a default gateway. On the receipt of a client packet, the dispatcher selects a target server and then rewrites the packet's destination IP address accordingly. The server's response packnation IP address accordingly. The server's response pack-
ets need to go through the dispatcher because it acts as the server's gateway. When the dispatcher receives the packets, it replaces their source IP addresses with the virtual IP address, making them look as if they were coming from the virtual server. For example, when a client packet arrives at the dispatch rives at the dispatcher, its destination address is the VIP, 141.200.10.1. The dispatcher decides the packet should be redirected to a server in the cluster, say Server 1 . Then the dispatcher changes the packet's destination address to 10.0.0.2. Server 1's response packets contain 10.0.0.2 as the source addresses. When they reach the dispatcher, their source addresses are replaced with the VIP. Due to the modification of the IP head, the checksum needs to be calculated twice.

TCP splicing is a layer-7 implementation (Cohen, Rangarajan, and Slye, 1999). It inserts a special TCP splicing component in between the network and the MAC layers. When the dispatcher is contacted by a client to establish a TCP connection, it accepts the request instead of rerouting it immediately to a server. The dispatcher then intercepts the client's subsequent requests. After that, the dispatcher selects a destination server and establishes another TCP connection with the server. Once both connections are set up, the TCP splicing component transfers all the requests between the two connections by altering their headers accordingly (source and destination IP addresses, IP and TCP header checksums, and other fields). Therefore, the request and response packets look as if they were being transferred in a single TCP connection. Figure 4 presents the detail of the routing mechanism by assuming a client with IP address 200.0.0.1 wants to access http://www.xyz.com. The client sends a connection request to the Web cluster (step 1). The dispatcher gets this request and establishes a connection with the client (step 2). After getting the client's data request, the dispatcher chooses a server, say Server 1, to handle the request and then splices the two connections (step 3). On the receipt of a client request with a source address of 200.0.0.1 and a destination of 141.200.10.1, the TCP splicing component modifies its source and destination addresses to 200.0.0.1 and 10.0.0.2, respectively (step 4).
After modification, the request is sent to the Server 1 directly (step 5). Similarly, when it receives a response from Server 1 with a source of 10.0.0.2 and a destination of 200.0.0.1, the splicing component changes its source and destination to 141.200.10.1 and 200.0.0.1, respectively (step 6). The response is forwarded to the client in (s) (step 7). Consequently, TCP connection splicing avoids many protocol overheads at high-level network layers.

The dispatcher with two-way configuration is easy to implement by hardware and does not require modifications of existing environment. However, the dispatcher with a two-way configuration tends to be a performance with a two-way configuation tens to be a performance (Schound requests and outbound responses (Schroeder, Goddard, and Ramamurthy, 2000). This problem becomes even more severe when the responses are bandwidth-demanding, particularly in streaming services.

\section{One-Way Dispatcher Architecture}

Packet forwarding is a layer-4 implementation. It assumes that the dispatcher is located in the same LAN as the servers. They all share one virtual IP address. The dispatcher configures the VIP as its primary address and the servers use the VIP as their secondary address. The servers disable their address resolution protocol (ARP) so that the incoming packets can reach the dispatcher without conflict. On the receipt of a client packet, the dispatcher forwards it to a selected server on the LAN by changing its destination MAC address to that of the server. The receiving server processes the client's packets and sends the response packets to the client directly, without the intervention of the dispatcher. For example, assume there is a tination of 141.200.10.1 arrives and the dispatcher selects a server, say Server 1, to respond to the packet. The dispatcher changes its destination MAC to 12:34:56:78:90:bb. Since all response packets use the VIP as their source, the service transparency is retained.

Packet tunneling is another layer-4 implementation based on IP encapsulation on the dispatcher and the servers (Simpson \& Daydreamer, 1995). IP encapsulation is to wrap each IP datagram within another IP datagram as payload, thus providing a method of redirecting the IP datagram to a destination different from the original one. In this approach, the IP addresses of the dispatcher and the servers should be configured in the same way as in the packet forwarding mechanism. After deciding a server for each incoming datagram, the dispatcher encapsulates the datagram via IP encapsulation; the source and destination of the outer header are the VIP and the selected server IP address, respectively. Then the new datagram is forwarded to the selected server. The target server unwraps the datagram and gets the original datagram. Because the server shares the VIP with the dispatcher, the original IP datagram is delivered to the server through its private (secondary) address. The packet tunneling approach requires IP encapsulation to be supported in the dispatcher and all the servers.

TCP connection hop is a software-based proprietary solution by Resonate (Resonate, 2001). It combines the advantages of IP encapsulation and TCP splicing. Its key 


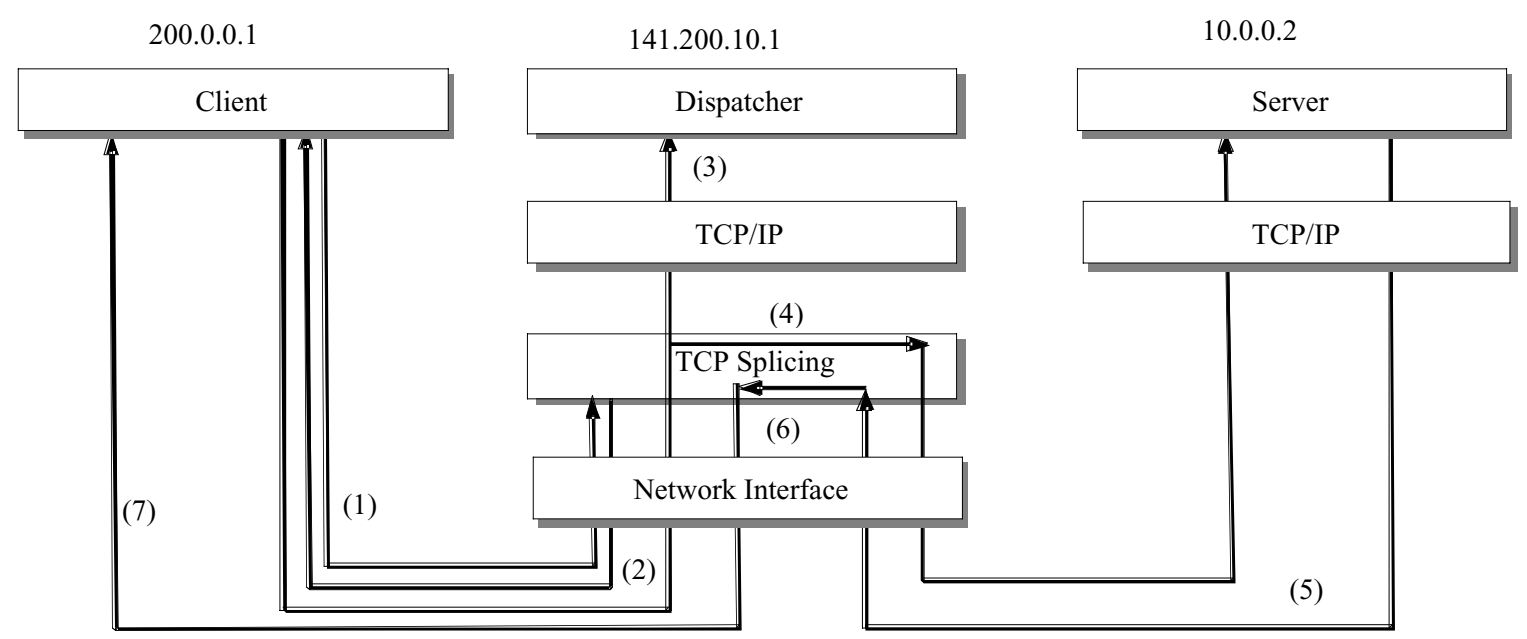

Figure 4: TCP splicing architecture.

component, resonate exchange protocol (RXP) operating between the network driver and TCP/IP stack, needs to be installed in the dispatcher and all the servers. When the RXP receives an IP datagram, it selects a server based on its load-balancing policy and encapsulates the original datagram in an RXP datagram for the target server. The RXP header of the RXP datagram is stripped off by the RXP component in the target server. The original dataRXP component in the target server. The original data-
gram travels up the TCP/IP stack and is processed as if it came directly from the client. Since the server shares VIP with the dispatcher, it replies directly to the client.

TCP handoff is a layer-7 implementation (Pai et al. 1998). Figure 5 depicts the architecture of the TCP handoff approach. The dispatcher and the servers are modified to put the TCP handoff protocol on top of the TCP/IP stack. put the TCP handoff protocol on top of the TCP/IP stack.
As an example, we assume that a client wants to access the Internet service. The client sends a connection request to the dispatcher; the request flows up the TCP/IP stack, and arrives at the dispatcher (step 1). The dispatcher accepts this request and establishes a connection with the client (step 2). The clien spatcher (step 3). The dispatcher receives the data request; makes a server selection decision based on the request content; and hands the connection to the target server using the handoff protocol (step 4). The server takes over the established connection (step 5). The server's responses are returned to the client directly as if it came from the virtual server (step 6). For acknowledgment packets, a forwarding module located between the network interface driver and the TCP/IP stack is used (step 7). The module first checks whether an acknowledgment packet should be forwarded. If so, the packet is modified and sent to the appropriate server without traversing the TCP/IP stack.

Note that both the one-way and two-way implementations of a dispatcher define load-balancing mechanisms and leave load-balancing policies open. In the following, different policies will be reviewed.

\section{State-Blind Load Balancing}

As in the DNS-based approaches, both the round-robin and randomized assignment policies can be applied for load balancing in the dispatcher. Albeit simple, they overcome the limitation of the DNS-based implementations. The state-blind policies overlook the client request-specific information and server dynamic states. This makes these approaches not very appealing in practice.

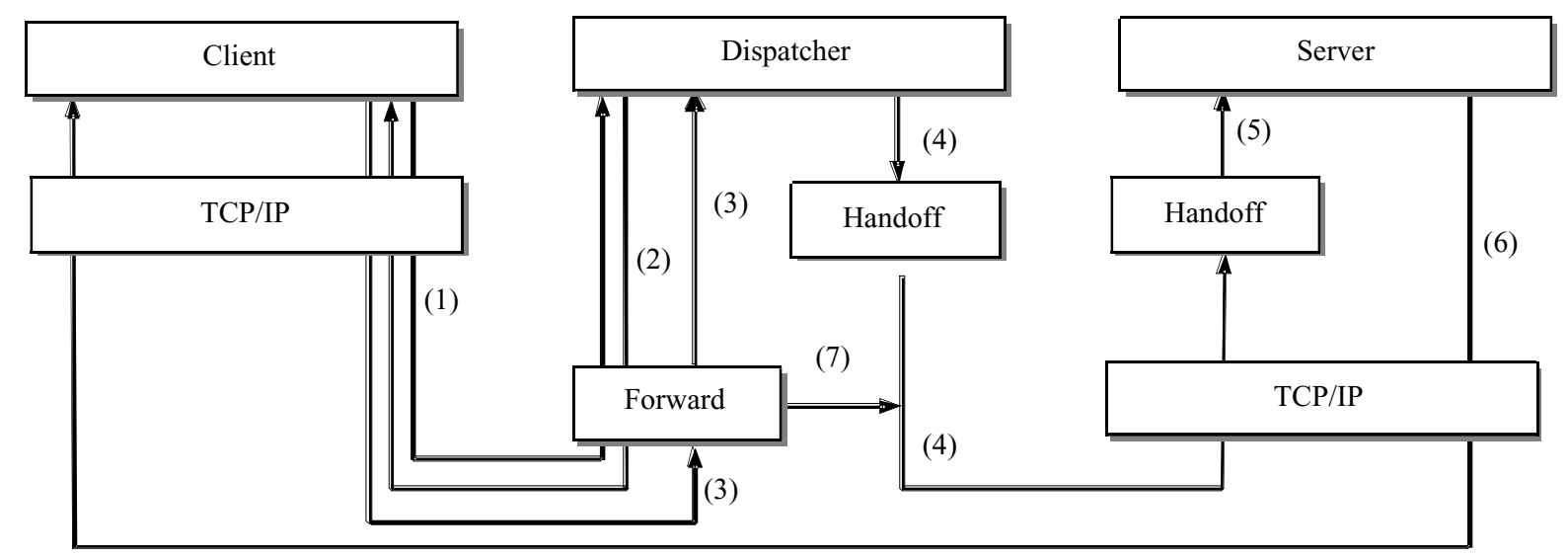

Figure 5: TCP handoff architecture. 
Client State-Aware Load Balancing

State-blind load-balancing approaches treat client requests independently. However, the client's requests are dependent on many Internet services (i.e. e-commerce). That is, these requests must be forwarded to the same server to preserve the dependent information, such as the content of a client's shopping cart, between requests. This required coordination in the process of related requests is called client affinity. One approach to keeping client affinity is to use the SSL session ID to ensure it. SSL runs on top of TCP and uses the session ID to uniquely identify each session. Therefore, the dispatcher can achieve client affinity by forwarding the packets with the same session ID to the same server. Some other methods, such as cookie, can also be used to keep client affinity (Kopparapu, 2002).

\section{Server State-Aware Load Balancing}

Server-side state information, like dynamic workload in terms of active connections, response time, etc., can be considered to improve the performance of load balancing.

The LocalDirector (Cisco Systems, n.d.b) uses a leastconnection policy to dispatch incoming client requests to the servers with the least active connections. Weighted least-connection policy takes the number of active connections and the servers' capacities into consideration. It is the same as that used in DNS-based approaches. The fastest response policy selects the server with the least response time.

\section{Client and Server State-Aware Load Balancing}

While the server state-aware load-balancing approaches consider the state information of servers, it is also necessary to take into account the client state information and combine them together. Except for the source IP address and TCP port number, the dispatcher can get more information from client requests; for example, it can get the URL information and the request content. Moreover, the client characteristic is also useful for load balancing.

Locality-aware request distribution (LARD) (Pai et al., 1998) makes server selection based on the client request content. It considers not only the load balancing but also content. It considers not only the load balancing but also
the cache hit rate. For example, requests are categorized into content types, such as A, B, and C. For requests for content type A, they are dispatched to Server 1, while requests for contents of types $\mathrm{B}$ and $\mathrm{C}$ are dispatched to Server 2. Hence, the cache system of Server 1 is mainly used by content of type A while the cache system of Server 2 is occupied by contents of types B and C. Consequently, both servers' cache hit rates increase, which in turn improves the system performance in terms of response time. Meanwhile, the dispatcher limits the number of accepted requests. It hence has the flexibility to distribute the load among all servers. The requests for the same type of contents are dispatched to the same server when the server is nonoverloaded. Otherwise, the requests are dispatched to the server with the least active connections.

An extension of LARD that reduces the overhead of the dispatcher is scalable content-aware request distribution (Aron, Sanders, \& Druschel, 2000). The client requests are redirected to different servers by a simple scheme, such as DNS-based round robin. Their request contents are inspected at individual servers and sent back to the dispatcher. The dispatcher uses the same policy as LARD to determine the most appropriate server.

The size interval task assignment with variable load (SITA-V) (Crovella, Harchol-Balter, \& Murta, 1998) is based on that the distribution of the client request sizes is heavy-tailed with infinite variance. SITA-V assigns requests with small sizes to the servers with lighter workquests with small sizes to the servers with lighter work-
load. It improves the ratio of the requests' waiting times to their service times. This can improve the user-perceived performance.

\section{Server Cooperative Load Balancing}

In general, cooperative load-balancing approaches deploy a two-level request routing mechanism. Client requests are initially assigned by the authoritative DNS server to target servers. It is followed by a possible redirection from the target server to others for load balancing. That is, based on some load-balancing policies, all servers are allowed to participate in the process of request distribution. These servers can be locally distributed or geographically distributed. Since all servers can decide which is the most appropriate server, there is no centralized point of becoming a potential performance bottleneck. Note that these schemes assume that the client requests can reach different servers through broadcasting or DNS-based round robin. Also, different load-balancing policies can be applied to these implementations.

\section{Request Redirection Mechanisms}

The redirection mechanisms used in server cooperative load balancing include IP filtering, HTTP redirection, URL rewriting packet rewriting and IP tunneling. The last one is the same as IP tunneling in the dispatcher-based approaches. IP filtering only accepts wanted packets. The IP filtering component operates between the network interface driver and TCP/IP stack. An incoming packet is broadcasted to all servers. When it arrives at the IP filtering component, the component decides whether it should accept the packet based on the load-balancing policy. If so, the packet is forwarded up to the TCP/IP stack for further processing.

HTTP redirection makes use of the HTTP redirection provided in HTTP protocols (Fielding et al., 1999). A client sends a request to a server. Then the server can reply to it with another server's address according to its loadbalancing policy and set the status code for redirection. By checking this status code, the client sends new requests to the selected server, if needed. For example, a client sends a request to Server 1. The server decides to let Server 2 process this request. It replies to the client with status code 301 ("Moved Permanently") or 302 ("Moved Temporarily") and sets Server 2's address as the new location. The client checks the status code and sends a new request to Server 2.

$U R L$ rewriting is based on dynamic URL generations. When a client requests a Web page, the URLs contained in this page are generated dynamically according to the server's load-balancing policy. These URLs point to different servers. Consequently, requests for these URLs are directed to different servers. For example, 
http://141.200.10.1/document.pdf is a link inside http:// www.xyz.com/index.html. When a client accesses this index page, the server can replace it with http:// 10.0.0.2/document.pdf before responding to the client. Thus, if client accesses this link, the request is sent to the new location.

Distributed packet rewriting is similar to packet rewriting (Bestavros, Crovella, Liu, and Martin, 1998). When a packet reaches a server, the server uses a hash function to calculate the packet's destination by the use of the client's IP address and port number to determine to which server the packet should be redirected. Then the server rewrites the packet to redirect it to the selected server. However for fragmented packets, since only the first fragment contains the required the required IP and port number, the rest of the packets could be misrouted.

\section{Load-Balancing Approaches}

Cooperative load-balancing approaches must have the knowledge about the servers. They are different in what kind of client state information is taken into account in server selection.

Network load balancing (Microsoft, 2000) makes use of IP filtering. When a packet arrives, an IP filtering component uses a randomization function to calculate the server priority based on the client's IP address, port number, and other state information. The server with highest priority accepts the packet and forwards the packet to the upper TCP/IP stack while other servers discard it. It also ensures TCP/IP stack while other servers discard it. It also ensures be mapped to the same server. However, the client's IP addresses and port numbers statistically depend on whether the client is residing behind proxy servers or other NAT devices. When client affinity is enabled, all clients from that domain are processed by one server and load balancing is defeated.

A distributed cooperative Web server (DCWS) uses the URL rewriting to implement its load-balancing policy (Baker \& Moon, 1999). Each server maintains the information of all pages in a local document graph. The graph consists of a set of tuples that store the page size, hit rate, and other information. When the client requests a Web page, the hyperlinks inside the page are dynamically genpage, the hyperlinks inside the page are dynamically gen-
erated based on their loads (i.e., hit rates) and the server's load. The main disadvantage of this approach is the calculation overhead of dynamically generated hyperlinks and the reconstruction of related pages.

A scalable Web server (SWEB) uses the HTTP redirection to distribute load across servers (Andersen, Yang Holmedahl, \& Ibarra, 1995). On the receipt of a client request, SWEB selects a server with least response time to direct the request. The calculation of the response time is on the basis of several parameters: redirection latency data reading time, CPU time, and network delay.

Workload-aware request distribution (WARD) uses a ward-analysis algorithm to determine the frequently requested contents, so-called base, that can be fitted into the cluster's memory (Cherkasova \& Karlsson, 2001). These contents are further partitioned into two groups, core and part, according to their sizes and frequencies of requests. The core contents include the most frequently accessed objects and is replicated and placed on the cache of al servers. The part contents are divided among servers in a balanced manner using hit rates and object sizes. These partitions are called local and remote partition. On the receipt of a client request, a server checks the request content. If the requested content is not in the base partition it is served locally. Otherwise, if the requested object belongs to core or local partition, it is served locally. In other cases, it is handed off to an appropriate server. WARD can reduce the request redirection number and improve the mean response time of client requests.

\section{NETWORK-SIDE LOAD BALANCING}

For scalability and availability, giant Internet services are often run on more than one geographical site on the Internet. Network-side load balancing is to distribute requests between different sites for balancing the workload of the sites and reducing the service transmission delay on the network. The geographically distributed servers can be organized in different ways. This section reviews two most important structures: caching proxy network and content delivery network , and their related load-balancing strategies.

\section{Client State-Aware Load Balancing: \\ Caching Proxy Network}

Web caching takes advantage of the temporal locality in requests for reducing the network load and access latency. Web caching proxies are the intermediate servers between clients and servers that act as agents representing the server to the client and the client to the server. As shown in Figure 6, Web caching proxies, including client-side forward caching proxies, network-side forward and reverse caching proxies, and server-side caching proxies, form a network. Forward caching proxies forward a client's request to the Web cluster, cache the response, and deliver the response to the client. These proxies are normally controlled by the clients or clients' network providers. Reverse caching proxies return requested content either from their caches or after fetching the content from its origin servers. Furthermore, reverse caching proxies are under the same administrative control as the Web cluster. By serving clients' requests from the proxies, the proxy network reduces the client-perceived network transmission latency, saves network bandwidth, and offloads the traffic of origin servers.

The caching proxy network can be organized as a hierarchical or mesh structure. In the hierarchical structure, low-level caching proxies close to the clients forward requests to a high-level proxy closer to the origin server i they cannot satisfy the requests. The solid arrows in Figure 6 represent a chain of proxies, from the lowest client forward proxy to the highest server proxy. The hierarchical proxy structure is an extension of computer memory hierarchy. It is simple to implement, but costly in space because a highly demanded Web object may be stored in many low-level cache proxies. By contrast, the mesh (cooperative) structure of caching proxy servers allows a forward proxy to communicate with its predetermined peers using an intercache protocol like Internet cache protocol (Wessels \& Claffy, 1997). If a caching proxy cannot find a valid response in its cache, it searches its peers. If they do, 


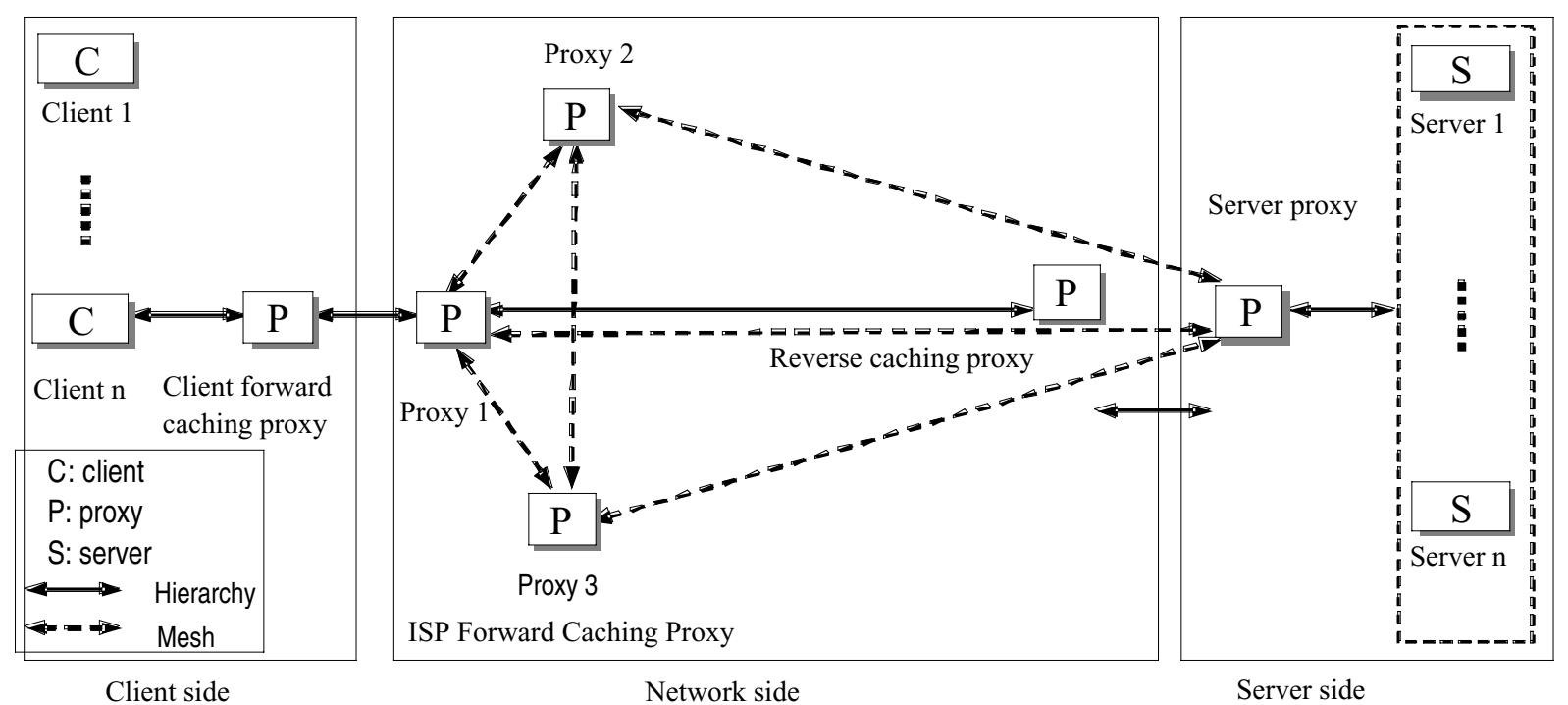

Figure 6: Architecture of a caching proxy network.

the request is directed to the first responding proxy. Otherwise, the proxy contacts the origin server for the response. The mesh structure reduces redundant copies in caching proxies at the cost of intercache communication. The hierarchical and mesh structures can be used in combination. For instance, in Figure 6, Proxy 1 can contact Proxies 2 and 3 when it is unable to satisfy an incoming client request. If neither Proxy 2 nor Proxy 3 has a valid response, the request is forwarded to the origin server through its reverse caching proxies and server-side proxy.

\section{Client and Server State-Aware Load Balancing: Content Delivery Network}

$C D N$ is an infrastructure for delivering content from locations closer than original content provider sites to clients by replication. CDN improves client-perceived performance of Internet services by serving client requests from CDN servers. It works similarly to caching proxy servers. However, CDN has some advantages (Rabinovich \& Spatscheck, 2001). First, CDN servers can be deployed around the network transparently, which is not always possible for caching proxies. The second is that service providers have full content control, such as content replication. For caching proxy servers, it is hard to control which content should be placed on which proxy servers. Another advantage is that CDN can improve performance for uncachable content, such as dynamic content and live streaming content. Finally, the content of Internet services can be placed on a CDN server before being accessed by clients.

There are two kinds of replication mode for CDN: full replication and partial replication. In full-replication mode, the origin sites are modified to use the authoritative DNS servers provided by CDN companies. The client requests can be delivered to CDN servers or be forwarded to the original sites. Partial replication needs modifications of the hyperlinks inside Web pages. For example, a hyperlink http://www.xyz.com/image.gif from XYZ company's homepage can be changed to http://www.cdn.com/www. xyz.com/image.gif. Therefore, when a client sends a request for this object, the host name needs to be resolved by CDN name servers. The products using such a DNS redirection mechanism include WebSphere from IBM (IBM, 2002) and EdgeSuite from Akamai (Akamai, 2002).

\section{DNS-Based Request Redirection}

For network-side load-balancing approaches, there are several mechanisms to redirect client requests to an appropriate geographically distributed server or site, such as DNS redi peering. As forementioned, DNS cannot effectively control server selection since such resolution can be satisfied by caches or name servers other than authoritative DNS servers. Assigning a small value to TTL may cause the DNS servers to bottleneck and increase client-perceived latency. To alleviate this problem, two-level DNS redirection is proposed (Leighton \& Lewin, 2000). On the top level, some DNS servers are used to resolve requests for higher-level domain names such as .com or .org. On the bottom level, the DNS database is replicated or partitioned among a number of geographically distributed DNS servers. When receiving a resolution request, a top-level DNS server reTTL value without making the DNS servers overloaded. Another approach is to place fully replicated DNS servers on the edge of the Internet. Resolution requests are directed to the DNS server closest to the client.

Another issue with DNS-based request redirection is an originator problem. It is known that an authoritative DNS server receives a request from a client's local DNS server instead of the client directly. However, sometimes, the location of the client is quite different from the location of the client's local DNS server. For example, in AT\&T WorldNet, most clients use only two DNS servers no matter where they are. Therefore, the closest server for a local DNS server sometimes is a distant one for the client compared to other servers. 


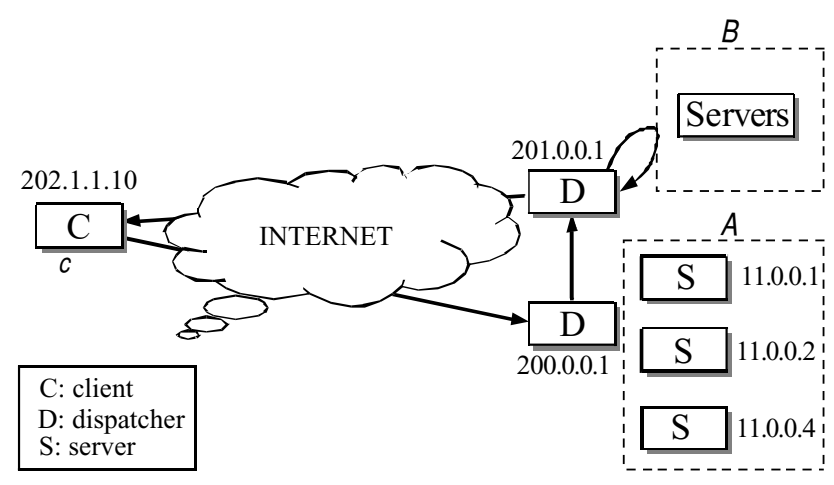

Figure 7: Network address translation and NAT peering.

\section{NAT Peering}

NAT peering is an implementation that can be used to redirect a client request from one site to another, avoiding the limitations of DNS-based redirection. The purpose of NAT is to provide a solution for IP address depletion. It provides a way to translate public IP addresses and TCP provides a way to translate public IP addresses and IP addresses and TCP ports. The main adports to private IP addresses and TCP ports. The main ad-
vantage of this approach is that it can be installed without vantage of this approach is that it
modifications of hosts or routers.

Figure 7 presents an illustration of NAT peering for request redirection between different sites or servers. As an quample, a client $c$ is on the public IP address 200.0.0.1. The NAT device in local area network $A$ receives the request and selects an appropriate site or server based on its load-balancing policy Assuming $B$ is selected, the request's destination address is changed accordingly while its source IP address is preserved. Therefore, the response is sent directly back to client $c$ while its source address is changed to A's IP address 200.0.0.1. Consequently, all client requests are still sent to its original destination $A$. NAT peering is widely supported by such switches as Cisco CSS11000 (Cisco System, 2002).

\section{Case Studies}

Akamai CDN. By using more than 12,000 servers in over 1,000 networks, Akamai's distributed content delivery system fights service bottlenecks and shut-downs by delivering content from the Internet's edge (Dilley et al., 2002). To handle traffic bursts, Akamai's infrastructure directs client requests to appropriate servers as well as additional servers. When receiving a client's resolution request, the Akamai CDN name server determines the target server Akamai CDN name server determines the target server cally, to determine the target server, it takes many metrics into account, such as network topology, bandwidth, and server's load. Moreover, the target server should have the requested content. For example, a media stream request should not be directed to a server that handles only HTTP. To prevent a server from overloading, a CDN's only HTTP. To prevent a server from overloading, a CDN's
load-balancing system must monitor the state of services, and their servers and networks. Akamai uses a threshold method for this purpose. All sites periodically report their service states and site load information to a monitoring application. When the load of a site exceeds a predefined threshold, mor quests. If the load exceeds another higher threshold, the site is excluded from the available site list until it is back to the threshold.

Enterprise $\mathrm{CDN}$ and examples. Large-scale Internet services deploy and administer their own enterprise CDNs. They use multiple levels of load balancing to distribute client requests for a single giant Internet service. At the top level, the services distribute their servers geographically to offer higher availability and better performance. At this level, services can adopt various request redirection mechanisms to distribute client requests to an appropriate site. The site selection can take into account the site's load and network's traffic information. After receiving a client request, the front-end, which can be a dispatcher, selects an appropriate server from local servers. At this level, all server-side load-balancing approaches can be deployed.

The Web site of the 1998 Olympic Winter Games is a good example of enterprise CDNs that IBM developed (Iyengar, Challenger, Dias, \& Dantzig. 2000). It supported nearly 635 million requests over the 16 days of the game and a peak rate of 114,414 hits in a single minute around the time of the women's freestyle figure skating. For scalability and availability, Web servers were placed in four different global wide sites. Client requests to http://www.nagano.olympic.org were routed from their local ISP into the IBM global services network (IGS), and IGS routers forwarded the requests to the server site geIGS routers forwarded the requests to the server site ge-
ographically nearest to the client. Each site had an installation of one SP-2 machine configured with 10 R6000 uniprocessors and an 8-way symmetric multiprocessor and had 12 open IP addresses. Load balancing at each site was conducted in a hierarchical way. At the top level, two routers acted as the entry points of each site. One is the primary point and another one is a backup. Between the primary point and another one is a backup. Between the
routers and the Web servers were four IBM network dis-
patchers (NDs) for load balancing. Each of these four NDs patchers (NDs) for load balancing. Each of these four NDs
was the primary source of 3 of the 12 IP addresses and the secondary source for two other addresses. These NDs were assigned to different values based on whether they were the primary or secondary source for an IP address. Secondary addresses for a ND were assigned a higher value. Therefore, the routers delivered incoming requests to the ND with the lowest value. On the receipt of the request the ND distributed it to a server based on the servers' load.

Three more examples of large-scale representative Internet services on their own CDNs were recently surveyed (Oppenheimer \& Patterson, 2002). To keep the services' identities confidential, the authors used the names of "Online," "Content," and "ReadMostly" to refer to an online service/Internet portal, a global content-hosting service, and a high- traffic Internet service with a very high readto-write ratio, respectively. At the survey time, both the Online and ReadMostly services supported around a hundred million hits per day and the Content service supported around 7 million hits per day. The Online service used around 500 servers at two data centers, the Conten 500 servers at four data centers, and the ReadMostly more than 2000 servers at four data centers. They generally use policies that take into account servers' load and availability to distribute client requests. The Online service provided its clients with an up-to-date list of appropriate 
servers for their selection. In the Content service, each two of its four sites worked in redundant pairs. Its clients need to install a software package that points to one primary and one backup site of the content service. The ReadMostly service used DNS-based load-balancing strategies based on its sites' load and health information. Among locally distributed servers, round-robin DNS servers or lacally distributed servers, round-robin DNS servers or the least loaded server.

Finally, we note that $\mathrm{CDN}$ was originally proposed for fast delivery of media-rich contents. For CDN to deliver live streaming media, there are some challenges because the media cannot be stored in advance on CDN servers. IP multicast or application-level multicast are used to distribute streaming media to CDN servers. For IP multicast, a multicast group is created by the origin server before the stream is distributed. A CDN server joins or leaves the group when it receives the first client request or finishes the last client request. Application-level multicast is based on a distribution tree of intermediate servers that makes use of IP unicast to deliver stream from the root (origin server) to the leaves (CDN servers).

\section{CLIENT-SIDE LOAD BALANCING}

Client-side load balancing mainly refers to load distribution between clients and servers. In addition, some services running on several servers are not transparent to their clients and the server selection is left to clients. We classify this type of client-guided load distribution as client-side load-balancing strategies, as well. In concept, client-side approaches can get as much client state information as they want. Thus, we classify these approaches in terms of whether they take server state information into account.

\section{Server State-Blind Load Balancing}

Client-side caches, like browsers' caches, can be constructed as an efficient and scalable peer-to-peer Web cache system for reducing servers' load by delivering requested content from this system (Xiao, Zhang, \& Xu. 2002). This approach assumes that the communication within a local area network is much faster than that between the clients and origin servers. These caches are managed by middleware components, which function as proxies for client requests, installed in all clients. When a client wants to access an Internet service, it first issues a request to the component within the same host. If the requested content is not cacheable, the component forwards the request to the origin servers. Otherwise, the component communicates with components running on other hosts to search the requested content. If a valid content is not present there, the request is sent to the external servers and the content is stored in the clients' caches for future use.

\section{Server State-Aware Load Balancing}

Some software distribution sites use another approach to balance the load of their servers. Before letting a client download their software, they provide several available servers with some location information. Meanwhile, they also provide the servers' URLs, geographical locations, and domain information. It is up to the clients to decide where to download the software. As an example, from the homepage of Free Software Foundation, we can find a list of available mirror servers that includes their location information. A client can select the geographically closest ension of this approach is dynamic server selection (Crovella \& Carter, 1995). In this approach, the round-trip time is measured periodically. Based on the mean of four round-trip measurements, the server with the least average round-trip time is selected to send the client's requests.

Smart client determines an appropriate server on the basis of server-side state (Yoshikawa et al, 1997). When a client visits a site, it downloads an applet from the site. The applet retrieves some server state information, such as its workload, response time, network connectivity, and configurations. As a result, the applet selects a server based on the state information. One disadvantage of this approach is that the information exchanging between the servers and the applet increases the network traffic.

\section{SUMMARY}

Load balancing on the Internet is to distribute the workload generated by Internet services between the server, client, and network aspects for the objective of providing high-level quality of service. This chapter presents a classification of load-balancing strategies in three dimensions: where they are realized (client side, server side, or network side), what knowledge the strategies take into account in making decisions, and on which network layer they are implemented. Under the framework, state-of-the-art loadbalancing strategies in each category are reviewed, focusing on their mechanisms, polices, and interplay with existing network routing and service delivery infrastructure.

\section{ACKNOWLEDGMENT}

This material is based on work supported in part by the National Science Foundation under Grants CCR-9988266 and ACI-0203592. Any opinions, findings, and conclusions or recommendation expressed in this material are those of the authors and do not necessarily reflect the views of the National Science Foundation.

\section{GLOSSARY}

Caching proxy network A group of caching proxies that collaborate to reduce the network traffic and the origin server load by exploiting the temporal locality of client requests.

Client load balancing A kind of technology performed with the cooperation of clients and servers to distribute client request load of an Internet service betribute client request load

client-aware load balancing The distribution of client requests to the servers of a Web cluster based on information about the clients.

Content delivery network A network of geographically distributed servers delegated to deliver content for origin servers. 
Content-aware load balancing The distribution of client requests to the servers of a Web cluster based on the requested content (see content-blind load balancing).

Content-blind load balancing The distribution of client requests to the servers of a Web cluster without considering any information about the requested out considering any information about the
content (see content-aware load balancing).

DNS-based load balancing The reliance on DNS servers to distribute client requests between locally and globally distributed servers during the process of domain name resolutions of requests.

Layer-4 dispatcher A device that distributes the client requests at the TCP layer when it is contacted for es-
rayer-4 dispatcher A device that distributes the client tablishing a TCP connection.

Layer-7 dispatcher A device that distributes the requests at the application layer by establishing a TCP connection with a client per request and delaying the request distribution until the request content is examined.

Least loaded distribution A load-balancing policy that assigns client requests to the server in a Web cluster with least load, which can be measured as a function of active connections, CPU utilization, etc.

Load balancing dispatcher A switch in front of a Web cluster that receives the client requests and directs them to appropriate servers; also referred to as load switch or load balancer.

Network load balancing The distribution of client requests of an Internet service between servers across wide area networks to reduce the service access latency and increase the service availability.

One-way dispatcher A device that intercepts all client requests of a Web cluster and allows the cluster servers to respond to the requests directly.

Round-robin load distribution The assignment of client requests to the servers of a Web cluster in a cyclic manner whereby each server appears one or more times in a round.

Server cooperative load balancing A two-level request routing mechanism that allows an assigned client request to be redirected to another server.

Server load balancing The distribution of client requests of a Web cluster between the cluster servers so as to reduce access latency and increase service scalability and availability.

Two-way dispatcher A device that intercepts both client requests and server responses.

Virtual IP The only IP address of a Web cluster visible to clients; in practice, often the public IP address of the load-balancing dispatcher.

Web cluster A group of servers that work collectively to provide the same Internet services at a single location.

\section{CROSS REFERENCES}

See Web Quality of Service; Web Services.

\section{REFERENCES}

Akamai.(2002). EdgeSuite. Retrieved October 5, 2002, from http://www.akamai.com/en/html/services/ edgesuite.html
Andersen, D., Yang, T., Holmedahl, V., \& Ibarra, O. H. (1995). SWEB: Towards a scalable World Wide Web server on multicomputers. In Proceedings of 10th IEEE International Parallel Processing Symposium (pp. 850-856). Los Alamitos, CA: IEEE Computer Society Press.

Arlitt, M. F., Krishnamurthy, D., \& Rolia, J. (2001). Characterizing the scalability of a large Web-based shopping system. ACM Transaction on Internet Technology, 1(1), 44-69.

Arlitt, M. F., \& Williamson, C. L. (1997). Internet web servers: Workload characterization and performance implications. IEEE/ACM Transaction on Networking, 5(5), 631-645.

Aron, M., Sanders, D., \& Druschel, P. (2000). Scalable content-aware request distribution in cluster-based network servers. In Proceedings of 2000 USENIX Annual Technical Conference (pp. 323-336). Berkeley, CA: USENIX Accociation.

Baker, S. M., \& Moon, B. (1999). Distributed cooperative Web servers. In Proceedings of the 8th International World Wide Web Conference (pp. 1215-1229). New York: Elsevier Science.

Bestavros, A., Crovella, M., Liu, J., \& Martin, D. (1998). Distributed packet rewriting and its application to scalable server architectures. In Proceedings of 6th International Conference on Network Protocols. Los Alamitos, CA: IEEE Computer Society Press.

Cherkasova, L., \& Karlsson, M. (2001). Scalable web server cluster design with workload-aware equest distribution strategy WARD. In Proceedings of the 3rd International Workshop on Advanced Issues of E-Commerce and Web-Based Information Systems (pp. 212-221). Los Alamitos, CA: IEEE Computer Society Press.

Chesire, M., Wolman, A., Voelker, G. M., \& Levy, H. M (2001). Measurement and analysis of a streamingmedia workload. In Proceedings of the 3rd USENIX Symposium on Internet Technologies and Systems (US ITS) (pp. 1-12). Berkeley, CA: USENIX Accociation.

Cisco Systems. (2002). Cisco CSS11000. Retrieved October 5, 2002, from http://www.cisco.com/warp/public/ $\mathrm{cc} / \mathrm{pd} / \mathrm{si} / 11000 /$

Cisco System. (n.d.b). Cisco LocalDirector 400 Series. Retrieved October 5, 2002, from http://www.cisco.com/ warp/public/cc/pd/cxsr/400/index.shtml

Cohen, A., Rangarajan, S., \& Slye, H. (1999). On the performance of TCP-splicing for URL-aware redirction In Proceedings of the 2nd USENIX Symposium on Internet Technologies and Systems (USITS). Berkeley, CA: USENIX Accociation.

Colajanni, M., Yu, P. S., \& Cardellini, V. (1998). Dynamic load balancing in geographically distributed heterogeneous web servers. In Proceedings of the 18th IEEE International Conference on Distributed Computing Systems (pp. 295-302). Los Alamitos, CA: IEEE Computer Society Press.

Crovella, M. E., Harchol-Balter, M., \& Murta, C. D. (1998). Task assignment in a distributed system: Improving performance by unbalancing load. In Proceedings of ACM Sigmetrics Conference (pp. 268-269). New York: ACM Press. 
Crovella, M. E., \& Carter, R. L. (1995). Dynamic server selection in the Internet. In Proceedings of the 3rd IEEE workshop on the Architecture and Implementation of High Performance Communication Subsystems (HPCS'95) (pp. 158-162). Los Alamitos, CA: IEEE Computer Society Press.

Dilley, J., Maggs, B., Parikh, J., Prokop, H., Sitaraman, R., \& Weihl, B. (2002). Globally distributed content delivery. IEEE Internet Computing, 6(5), 50-58. Los Alamitos, CA: IEEE Computer Society Press.

Egevang, K., \& Francis, P. (1994). The IP network address translator (NAT) (RFC 1631). Network Working Group. Reston, VA: The Internet Society.

F5 Networks. (n.d.). 3-DNS Controller: Make the most of your network. Retrieved October 5, 2002, from http:// www.f5.com/f5products/3dns/

Fielding, R., Gettys, J., Mogul, J., Frystyk, H., Masinter, L., Leach, P., et al. (1999). Hypertext transfer protocol—HTTP/1.1 (RFC 2616). Network Working Group. Reston, VA: The Internet Society.

Harchol-Balter, M., Crovella, M. E., \& Murta, C. D. (1999). On choosing a task assignment policy for a distributed server system. International Journal of Parallel and Distributed Computing, 5(2), 204-228. New York: Elsevier Academic Press.

Hennessy, J. L., \& Patterson, D. A. (2003). Computer architecture: A quantitative approach (3rd ed.). San Mateo, CA: Morgan Kaufmann.

IBM. (2002). IBM WebSphere software platform. Retrieved October 5, 2002, from http://www.ibm.com/ websphere

Iyengar, A., Challenger, J., Dias, D., \& Dantzig, P. (2000). High-performance Web site design techniques. IEEE Internet Computing 4(2), 17-26. Los Alamitos, CA: Internet Computing, 4(2), $17-26$.

Kopparapu, C. (2002). Load balancing servers, firewalls, and caches. New York: Wiley.

Kwan, T. T., McGrath, R. E., \& Reed, D. A. (1995). NCSA's World Wide Web server: Design and performance. IEEE Computer, 28(11), 68-74. Los Alamitos, CA: IEEE Computer Society Press.

Leighton, T., \& Lewin, D. (2000). Global document hosting system utilizing embedded content distributed ghost servers (International Publication WO 00/04458). World Intellectual Property Organization.

Menasce, D. A., Almeida, V. A. F., Fonseca, R., \& Mendes, M. A. (1999). A methodology for workload characterization of e-commerce sites. In Proceedings of the ACM Conference on Electronic Commerce (pp. 119$A C M$
$128)$.
Microsoft. (2000). Network load balancing technical overview (white paper). Retrieved October 5, 2002, from http://www.microsoft.com/windows2000/ techinfo/howitworks/cluster/nlb.asp

Oppenheimer, D., \& Patterson, D. A. (2002). Architecture and dependability of large-scale Internet services. (1) IEEE Internet Computing, 6(5), 41-49. Los Alamitos, CA: IEEE Computer Society Press.

Padmanabhan, V. N., \& Qui, L. (2000). The content and access dynamics of a busy Web site: Findings and implications. In Proceedings of the ACM SIGCOMM (pp. 111-123). New York: ACM Press.

Pai, V. S., Aron, M., Banga, G., Svendsen, M., Druschel, P., Zwaenepoel, W., et al. (1998). Locality-aware request distribution in cluster-based network servers. In Proceedings of the 8th ACM International Conference on Architectural Support for Programming Languages and Operating Systems (pp. 205-216). New York: ACM Press.

Rabinovich, M., \& Spatscheck, O. (2001). Web caching and replication. Reading, MA: Addison-Wesley.

Resonate. (2001, April). TCP connection hop (white paper). Retrieved October 5, 2002, from http://www. resonate.com/solutions/literature/iwp_cd_tcp_connect_ hop.php

Schroeder, T., Goddard, S., \& Ramamurthy, B. (2000). Scalable Web server clustering technologies. IEEE Network, 14(3), 38-45.

Simpson, W., \& Daydreamer. (1995). IP in IP tunneling (RFC 1853). Network Working Group. Reston, VA: The Internet Society.

Wessels, D., \& Claffy, K. (1997). Internet cache protocol $(I C P)$, version 2 (RFC 2186). Network Working Group. Reston, VA: The Internet Society.

Wolf, J. L., \& Yu, P. S. (2001). On balancing the load in clustered Web farm. ACM Transaction on Internet Technology, 1(2), 231-261. New York: ACM Press.

Xiao, L., Zhang, X., \& Xu, Z. (2002). On reliable and scalable peer-to-peer Web document sharing. In Proceedings of 2002 IEEE International Parallel and Distributed Processing Symposium. Los Alamitos, CA: IEEE Computer Society Press.

Xu, C.-Z., \& Lau, F. (1997). Load balancing in parallel computers: Theory and practice. Dordrecht: Kluwer Academic.

Yoshikawa, C., Chun, B., Eastham, P., Vahdat, A., Anderson, T., \& Culler, D. (1997). Using smart clients to build scalable services. In Proceedings of the USENIX Annual Technical Conference (pp. 105-117). Berkeley, CA: nual Technical Confere
USENIX Accociation. 